\title{
Antiobesity activity of Acer tegmentosum Maxim on 3T3-L1 preadipocyte and high-fat diet-induced obese rats
}

\section{Hang-Hee Cho}

Gyeongsang National University

\section{Soo-Jung Lee}

Gyeongsang National University

\section{Sung-Ho Kim}

Gyeongsang National University

\section{Sun-Hee Jang}

Gyeongsang National University

\section{Chungkil Won}

Gyeongsang National University

\section{Hong-Duck Kim}

New York Medical College

\section{Tae Hoon Kim}

Daegu University

Jae-Hyeon Cho ( $\square$ jaehcho@gnu.ac.kr)

Gyeongsang National University

\section{Research}

Keywords: Acer tegmentosum Maxim, High-fat diet, 3T3-L1 cells, Adipocyte differentiation, Obesity

Posted Date: February 24th, 2020

DOl: https://doi.org/10.21203/rs.2.24283/v1

License: (1) This work is licensed under a Creative Commons Attribution 4.0 International License. Read Full License 


\section{Abstract}

Background: The aim of this study was to investigate the effect of Acer tegmentosum Maxim (ATM) on adipocyte differentiation in 3T3-L1 adipocyte-derived cells and anti-obesity properties in high fat diet (HFD)-induced obese rats. Methods: 3T3-L1 adipocytes and HFD-induced obese rats were treated with ATM, and its effect on gene expression was analyzed using RT-PCR and Western blotting experiments. Results: Cellular lipid contents in DMI (dexamethasone, 3-isobutyl-1-methylxanthine, and insulin mixture)treated cells increased, while ATM treatment caused a significant reduction in lipid accumulation in differentiated 3T3-L1 cells. ATM caused inhibition of adipogenesis via down-regulation of the CCAAT/enhancer binding protein $\beta$ (C/EBP $\beta), C / E B P a$, and peroxisome proliferator-activated receptor $Y$ (PPARY) expressions in 3T3-L1 cells. Moreover, treatment with ATM caused a decrease in the expressions of adipocyte-specific genes, such as adipocyte fatty acid-binding protein-2 (aP2), fatty acid synthase (FAS), and lipoprotein lipase (LPL), compared with DMl-stimulated adipocytes. In addition, phosphorylation levels of protein kinase B (Akt) and its downstream substrate, glycogen synthase kinase $3 \beta$ (GSK3 $\beta$ ), were significantly decreased by ATM treatment of 3T3-L1 adipocytes. Together, these results indicated that ATM caused inhibition of both adipocyte differentiation via suppression of the C/EBP family and PPARy expressions and the Akt signaling pathway in 3T3-L1 adipocytes. In the present study, we further investigated anti-obesity effects of ATM on HFD-induced obese rats. Rats fed with HFD demonstrated elevations in body weight gain, while the administration of ATM significantly reversed BW gains and adipose tissue weights in rats fed HFD. ATM supplementation also caused a decrease in the circulating triglyceride levels and total cholesterol levels and led to inhibition of lipid accumulation in the adipose tissues in HFD-induced obesity in rats. Furthermore, epididymal fat exhibited larger adipocytes in the HFD group, whereas the ATM-treated group was significantly smaller than that of HFD group. These results strongly demonstrate that ATM administration caused a reduction in adiposity via attenuation in adipose tissue mass and adipocyte size. Conclusion: These finding demonstrated that ATM exerted antiobesity effects through inhibition of adipocyte differentiation and adipogenesis, leading to a decrease in BW and fat tissue mass in HFD-induced obesity in rats.

\section{Background}

Obesity is a major risk factor of many metabolic disorders, including hyperlipidemia, type 2 diabetes, atherosclerosis, nonalcoholic fatty liver disease, and cardiovascular diseases [1]. Fat is properly defined as adipose tissue and is a biological caloric reservoir that expands in response to overnutrition and releases lipids in response to energy deficient conditions. Although adipose tissues are the major site for excess energy storage, excess adiposity and adipocyte dysfunction cause induction of increased levels of plasma free fatty acids and triglycerides (TGs), decreased levels of high-density lipoprotein (HDL), and abnormally low-density lipoprotein (LDL) composition in plasma and tissues, such as liver and muscle. These changes lead to pathological dysfunction of these tissues [2,3]. Also, it is important for an individual to maintain an adequate balance between energy accumulation and consumption in order to prevent and improve obesity. 
Adipose cell hyperplasia (cell number increase) and hypertrophy (cell size increase) might be an important factor in obesity development. Adipocyte differentiation and intracellular lipid accumulation are associated with the occurrence and development of obesity [4]. Adipogenesis is a differentiation process by which undifferentiated preadipocytes are converted to fully differentiated adipocytes. Thus, inhibition of adipogenesis and adipocyte differentiation from fibroblast-like preadipocytes into fully mature adipocytes in addition to identifying critical factors that regulate these processes is extremely important in an approach to primary obesity prevention and treatment.

The 3T3-L1 cell line is a well-established and widely used in vitro model of obesity for assessing adipocyte differentiation [5]. The differentiation process of 3T3-L1 cells into adipocytes follows a precisely ordered and temporal series of events regulated by a number of transcription factors and several signaling pathways for the development of the mature lipid-filled adipocytes $[6,7]$. During the differentiation process, the many key transcriptional factors that are involved, such as the CCAAT enhancer binding protein $\delta(C / E B P \delta)$ and $C / E B P \beta$, work in conjunction to induce peroxisome proliferatoractivated receptor $\mathrm{Y}$ (PPARY) and C/EBPa expressions [8]. C/EBP $\beta$ and C/EBPS are expressed comparatively early in adipogenesis; this event is an important factor for initiating the transcriptional cascades that culminate in the expression of two essential adipogenic factors, PPARY and C/EBPa [9]. PPARY and C/EBPa are critical transcription factors involved in adipogenesis and regulatin of the genes, such as lipoprotein lipase (LPL), adipocyte fatty acid-binding protein-2 (aP2), and fatty acid synthase (FAS) $[10,11]$, which are involved in adipogenesis and lipogenesis. Moreover, these transcription factors are also known to influence adipocyte differentiation in vivo.

The protein kinase B (Akt) signaling pathway plays a major role in adipocyte differentiation induced by a downstream function of insulin, and activation of the Akt pathway in 3T3-L1 preadipocytes contributes to adipocyte differentiation [12], whereas inactivation of the PI3K/Akt pathway inhibits adipogenesis [13]. Akt phosphorylates and regulates a number of substrates involved in a diverse array of biological process [14] and is essential for induction of PPARy expression [15]. Moreover, overexpression of constitutively active Akt causes an increase in glucose uptake and adipocyte differentiation in 3T3-L1 adipocytes [16]. In contrast, the RNAi-mediated decrease in Akt expression blocks 3T3-L1 cells differentiation [17]. Adipocyte differentiation and lipid accumulation is impaired in the absence of Akt, whereas Akt overexpression in Akt-deficient cells leads to lipid droplet accumulation [18].

Acer tegmentosum Maxim (ATM) is widely used as a traditional medicinal plant in Asia. A. tegmentosum has traditionally been used for treatment of various hepatic disorders, including hepatitis, cirrhosis, and liver cancer [19]. Phytochemical compounds isolated from A. tegmentosum has been reported to be one of the important sources of useful bioactive secondary metabolites, such as flavonoids, lignans, and phenolic compounds [20,21]. In addition, antioxidants, anti-inflammatory, and anti-lipogenic activities were also shown in biological investigations on ATM extracts [22, 23]. However, the anti-obesity effects of ATM are still poorly understood. 
In the present study, we examined the inhibitory effects of ATM on adipogenesis, its underlying molecular mechanisms in 3T3-L1 cells, and body weight reduction effects in high-fat diet (HFD)-induced obesity in rats.

\section{Materials And Methods}

\section{Preparation of ATM}

The Acer tegmentosum Maxim (ATM) stem was purchased from Jinju traditional herbal market (GyeongsangNam-do, Jinju, Korea). The dried and chopped twigs and inner barks of ATM was powdered and extracted with $70 \%$ ethanol at $60{ }^{\circ} \mathrm{C}$ for $72 \mathrm{~h}$. The extract was filtered, dried under reduced pressure, and concentrated by an evaporator to obtain an ethanol-soluble fraction. Lyophilized ATM powder was dissolved in with dimethyl sulfoxide (DMSO, Sigma-Aldrich) at a concentration of $200 \mathrm{mg} / \mathrm{mL}$ and stored at $-20^{\circ} \mathrm{C}$ for further use.

\section{Cell culture}

3T3-L1 preadipocytes obtained from the Korean Cell Line Bank (Seoul, Korea) were cultured in Dulbecco's modified eagle high-glucose medium (DMEM) supplemented with $10 \%$ calf serum at $37^{\circ} \mathrm{C}$ in a humidified atmosphere of $5 \% \mathrm{CO} 2$. To induce differentiation, 2-day post-confluent preadipocytes (designated day 0) were cultured induced with DMI differentiation medium (0.5 mM 3-isobutyl-1methylxanthine, $1 \mu \mathrm{M}$ dexamethasone, and $0.5 \mu \mathrm{g} / \mathrm{mL}$ insulin in DMEM containing $10 \% \mathrm{FBS})$. The 3isobutyl-1-methylxanthine (MIX), dexamethasone (DEX), Insulin, and Oil Red O were obtained from SigmaAldrich (St. Louis, MO, USA). The medium was changed every 2 days. ATM extracts were added to the culture medium of the adipocytes on day 0 . The cells were treated with 0,20 , or $60 \mu \mathrm{g} / \mathrm{mL}$ ATM extracts every day. After treatment with ATM for 4 and 8 days, the 3T3-L1 adipocytes were lysed for Western blot analysis. To analyze cell viability, the cytotoxicity of the ATM was evaluated using 3-(4, 5demethylthiazol-2-yl)-2, 5-diphenyltetrazolium bromide (MTT).

\section{Oil Red 0 staining and triglyceride assay}

After the induction of differentiation, cells were stained with Oil Red O (60\% isopropanol and $40 \%$ water), Briefly, the cells were gently washed with phosphate-buffered saline (PBS) and stained with filtered Oil Red $\mathrm{O}$ solution for $30 \mathrm{~min}$. After staining, the Oil Red $\mathrm{O}$ staining solution was removed, and the plates were rinsed with water and dried. The stained lipid droplets were viewed on an Olympus microscope (Tokyo, Japan). To analyze the content of the cellular triglycerides, the cells were washed with PBS, scraped into $200 \mu \mathrm{L}$ of PBS and sonicated for $1 \mathrm{~min}$. The lysates were assayed for their total triglyceride content using assay kits from Sigma-Aldrich (St. Louis, MO, USA) and for cellular protein using the BioRad protein assay (CA, USA). The results were expressed as $\mathrm{mg}$ of triglyceride per $\mathrm{mg}$ of cellular protein.

\section{RT-PCR}


Cellular RNA was extracted from 3T3-L1 adipocytes using the Trizol reagent (Invitrogen, CA, USA) according to the manufacturer's instructions. One microgram of total RNA was used for first strand cDNA synthesis with oligo (deoxythymidine) primers and Superscript II reverse transcriptase (Invitrogen, CA, USA). The target cDNA was amplified using the following primers: C/EBP $\beta, 5^{\prime}$ -

GACTACGCAACACACGTGTAACT-3' and 5'-CAAAACCAAAAACATCAACAACCC-3'; PPARY, 5'-

TTTTCAAGGGTGCCAGTTTC-3' and 5'-AATCCTTGGCCCTCTGAGAT-3'; C/EBPa, 5'-

TTACAACAGGCCAGGTTTCC-3' and 5'-GGCTGGCGACATACAGATCA-3'; $\beta$-actin (control), 5'-

GACAACGGCTCCGGCATGTGCAAAG-3' and 5'-TTCACGGTTGGCCTTAGGGTTCAG-3'. The amplification cycles included denaturation at $95^{\circ} \mathrm{C}$ for $50 \mathrm{sec}$, annealing at $55^{\circ} \mathrm{C}$ for $1 \mathrm{~min}$ and elongation at $72{ }^{\circ} \mathrm{C}$ for $50 \mathrm{sec}$. After 30 cycles, the PCR products were separated by electrophoresis on a 1.5\% agarose gel for 30 min at $100 \mathrm{~V}$. The gels were stained with $1 \mathrm{mg} / \mathrm{ml}$ ethidium bromide and visualized with UV light using the BIO-RAD Gel Doc image analysis software (BIO-RAD Laboratories Inc., CA, USA).

\section{Western blot analysis}

Western blotting was performed according to standard procedures. Briefly, cells were lysed in lysis buffer containing $50 \mathrm{mM}$ Tris- $\mathrm{HCl}$ (pH 8.0), 0.4\% Nonidet P-40, $120 \mathrm{mM} \mathrm{NaCl}, 1.5 \mathrm{mM} \mathrm{MgCl} 2,0.1 \%$ SDS, $2 \mathrm{mM}$ phenylmethylsulfonyl fluoride, $80 \mu \mathrm{g} / \mathrm{ml}$ leupeptin, $3 \mathrm{mM} \mathrm{NaF}$ and $1 \mathrm{mM}$ DTT. Cell lysates were separated by $10 \%$ SDS-polyacrylamide gel electrophoresis, transferred onto a polyvinylidene fluoride membrane (Amersham Pharmacia, England, UK), blocked with $5 \%$ skim milk and hybridized with primary antibodies. PPARy, C/EBP $\beta$, C/EBPa, aP2, FAS, Akt, phospho-Akt (p-Akt), phsopho-GSK3 $\beta$ (p-GSK3 $\beta$ ), and GSK3 $\beta$ antibody were from Cell Signaling and the monoclonal $\beta$-actin antibody was from Chemicon. HRP-labeled mouse anti-rabbit IgG were from Jackson ImmunoResearch. The Chemiluminescence kit was from Pierce (Rockford, IL). After incubation with horseradish-peroxidase-conjugated secondary antibody at room temperature, immunoreactive proteins were detected using a chemiluminescent ECL assay kit (Amersham Pharmacia, UK) according to the manufacturer's instructions.

\section{Animal experiments}

The study protocol was approved by the Ethic committee for Animal Experiments of Gyeongsang National University. All rats were handled according to the University Guidelines and the Animal Care Committee Guidelines of Gyeongsang National University (GNU-181231-R0066). 40 male SpragueDawley (SD) rats weighing approximately $180 \mathrm{~g}$ were purchased from the Central Lab. Animal Inc. (Seoul, Korea). Rats were acclimatized to the experimental facility for 1 week. All animals were kept under controlled temperature and on a $12 \mathrm{~h}$ light and $12 \mathrm{~h}$ dark cycle. The rats were divided into 3 groups of 10 and individually housed in polycarbonated cages in a room maintained at $22{ }^{\circ} \mathrm{C}$ and $55 \%$ relative humidity. All rats were allowed free access to their respective diets and drinking water for 5 weeks. Food intake was measured daily and body weight every two days. Obese rats were generated by feeding them a high fat diet (HFD). Normal diet group (ND) was maintained on a normal diet based on a commercial diet (\#55VXT0038, Samyang Co, Korea). HFD group (HFD) was fed an HFD based on a commercial diet 
(rodent diet with 60\% kcal fat, Research Diet, Korea). HFD plus ATM group (HFD+ATM) was fed an HFD, and ATM $(150 \mathrm{mg} / \mathrm{kg} \mathrm{BW})$ was administered through the gastrointestinal tract.

\section{Biochemical assays}

After 5 weeks on experimental diets, the rats were anesthetized, and the tissues were dissected and analyzed. The body weight and fatty tissue mass were measured with sensitivity limits of $0.1 \mathrm{~g}$ and 0.01 $\mathrm{g}$, respectively. The body weight index was calculated by dividing the weight $(\mathrm{g})$ by the square of body length (cm2). Blood was isolated from each rat, stored at $37^{\circ} \mathrm{C}$ for $30 \mathrm{~min}$, and centrifuged at $4000 \mathrm{x} \mathrm{g}$ at $4{ }^{\circ} \mathrm{C}$ for 10 min to obtain plasma. The epididymal fat pad and perirenal fat pad were excised, weighed and stored at $-20^{\circ} \mathrm{C}$ until assayed. The concentrations of plasma triglyceride (TG), total cholesterol (TC), and high-density lipoprotein (HDL)-cholesterol were assayed enzymatically using commercial kits (Asan phams, Co., Korea).

\section{Histological Analysis}

The epididymal fat pads and liver tissues was removed and fixed in $10 \%$ neutral buffered formalin. The fat pads and livers were subsequently embedded in paraffin, sectioned into $5 \mu \mathrm{m}$ sections (Leica, Wetzlar, Germany), and stained with hematoxylin-eosin for microscopic assessment (Olympus, Tokyo, Japan). Three different cross-sectional areas and their cell populations were calculated using an image analysis program (Image-Pro Plus 6.0).

\section{Statistical analysis}

All experiment results were reported as the means \pm SD and the experiments were repeated three $\sim$ four times. The differences between groups were determined using ANOVA and Duncan's multiple range tests. $P<0.05$ was usually considered as statistically significant.

\section{Results}

\section{Effect of ATM on intracellular lipid accumulation and TG content in 3T3-L1 cells}

The anti-adipogenic effects caused by ATM on preadipocyte differentiation into adipocytes were investigated. The degree of adipogenesis of 3T3-L1 cells was measured by the amount of lipid accumulation using lipid-specific staining with Oil Red $\mathrm{O}$ at eight days after the induction of adipocyte differentiation. Differentiated adipocytes were identified and characterized based on the number of lipid droplets, which are not detected in undifferentiated cells. The quantification of Oil Red 0 staining demonstrated that treatment with 20 to $60 \mu \mathrm{g} / \mathrm{mL}$ of ATM significantly attenuated adipocyte differentiation. ATM strongly affected cellular lipid accumulation, which was decreased by approximately $40 \%$ in a dose-dependent manner (Fig. 1A). Intracellular TG levels were also measured on day 4 or 8 of 3T3-L1 adipogenic differentiation. The cellular TG content significantly increased in the DMI-treated group during 3T3-L1 differentiation for four or eight days, while the addition of ATM into the differentiation medium strongly blocked TG accumulation (Fig. 1B). Together, the results from Oil Red 0 
staining and TG content assay demonstrated that ATM caused suppression of 3T3-L1 preadipocyte differentiation and decreased adipogenesis, intracellular lipid accumulation, and TG contents.

\section{Effect of ATM on cell viability}

The effects of ATM on cell viability were measured with the 3-(4,5 dimethylthiazol-2, 5 diphenyl) tetrazolium bromide (MTT) assay. 3T3-L1 cells were exposed to various concentrations $(20 \sim 60 \mu \mathrm{g} / \mathrm{mL})$ of ATM extracts for four or eight days. Results from the MTT assay showed that $24 \mathrm{~h}$ incubation of cells with the ATM extract produced no toxic effects on cell viability at concentrations up to $60 \mu \mathrm{g} / \mathrm{mL}$ (Fig. 1C). In this study, ATM concentrations in additional experiments were used up to $60 \mu \mathrm{g} / \mathrm{mL}$.

\section{Effect of ATM on the expression of adipogenic-specific genes during adipocyte differentiation}

Adipogenesis was accompanied by an increase in adipogenic-related transcription factors and adipocytespecific gene expression. In particular, $\mathrm{C} / \mathrm{EBP} \beta, \mathrm{C} / \mathrm{EBPa}$, and PPARy play a major role in adipogenesis. We examined whether ATM affected the mRNA levels related to expression of adopogenic transcription factors, such as C/EBPB, C/EBPa, and PPARy using reverse-transcriptase polymerase chain reaction (RT$P C R$ ) during adipocyte differentiation. As expected, the mRNA expression levels of C/EBP $\beta, C / E B P a$, and PPARY genes significantly increased in differentiated 3T3-L1 adipocytes (Fig. 2A). ATM treatment caused significant down-regulation of C/EBP $\beta, C / E B P a$, and PPARY mRNA expressions in differentiated 3T3-L1 adipocytes.

Furthermore, the effects of ATM on the protein expression of the adipogenic transcription factors was examined using Western blotting analysis. C/EBPB, C/EBPa, and PPARY protein expression levels decreased in a dose-dependent manner in response to ATM in the 3T3-L1 adipocytes compared with those of untreated control cells (Fig. 2B). Our data indicate that ATM induced inhibition of adipogenesis transcription factor expression with subsequent blocking of adipocyte differentiation.

\section{Effect of ATM on the expression of lipogenesis-specific genes during adipocyte differentiation}

Next, we investigated whether ATM could regulate the expression levels of lipogenesis-specific genes, such as FAS, LPL, and aP2. C/EBPa and PPARy regulated the expression of their target genes, such as FAS, LPL, and aP2, during adipogenesis since those genes play a crucial role in determining and maintaining the mature adipocyte phenotype. Differentiated 3T3-L1 adipocytes showed an increase in FAS, LPL, and aP2 expression levels, while treatment with ATM caused a significant, dose-dependent, down-regulation in the expression of these genes (Fig. 2C). These results indicate that ATM produces strong inhibition of lipid accumulation and causes a reduction in lipogenesis-specific gene expression levels in 3T3-L1 adipocytes.

\section{Effect of ATM on the regulation of Akt and GSK3 $\beta$ during adipocyte differentiation}

In order to investigate whether the Akt signaling pathway, a key regulator in adipogenesis, is activated by ATM during differentiation of 3T3-L1 cells, the phosphorylation level of Akt was examined after treatment 
with DMI alone or with DMI and ATM together. The results indicate that ATM treatment caused a significant and dose-dependent inhibition of phosphorylated Akt levels (Fig. 3A). Moreover, ATM caused a reduction in the level of phosphorylated GSK3 $\beta$, a downstream substrate of Akt (Fig. 3B). These results demonstrate that activation of phosphorylated-Akt was inhibited by ATM; moreover, phosphorylation of GSK3 $\beta$ during adipogenesis in 3T3-L1 cells was also inhibited.

\section{Effect of ATM on body weight and adipose tissue weight in HFD induced obesity rats}

We further investigated the effects of ATM on body weight and adiposity in rats fed an HFD for five weeks. After one week of adaption, the rats used for this study were randomly distributed into three groups of ten rats: (1) a normal diet (ND) group; (2) high fat diet (HFD) group; and (3) HFD group with ATM $(150 \mathrm{mg} / \mathrm{kg} /$ day; HFD+ATM). Body weight (BW) at five weeks significantly increased in rats in the HFD group compared with ND group, demonstrating that HFD fed rats developed into obese rats (Fig. $4 A)$. The weight gains in the group of rats fed with the ATM extract significantly decreased compared with the HFD group. However, there were no significant differences in the amount of food intake in the HFD group compared with that of the other groups during the experimental period. Thus, ATM-induced suppression of body weight gain did not depend on reduction of food and water intake. We also found that rats treated with ATM exhibited lower perirenal and epididymal fat mass compared to HFD group (Fig. 4B), demonstrating that the reduction in body weight gain was primarily due to reduced lipid accumulation in adipocytes.

\section{Effect of ATM on blood biochemical indexes in HFD-induced obese rats}

In order to further investigate the anti-obesity effects of ATM, we estimated the changes in serum TG, TC, and HDL-C levels in HFD-induced obesity in rats. As shown in Fig 5, TG and TC levels were significantly higher in the HFD-fed rat group compared with those in the ND group (Fig. 5A). However, TG and TC levels in the HFD group significantly decreased by $36 \%$ and $30 \%$, respectively, following ATM supplementation. Moreover, serum HDL-C levels in the ATM group significantly increased compared to those in the HFD group (Fig. 5A). Thus, the ATM extracts caused a significant decrease in serum TG and TC levels and increase in HDL-C levels.

\section{Effect of ATM on lipid accumulation in epidydymal adipose tissue}

Next, in order to investigate whether the epididymal fat mass reduction in the ATM-treated group led to a decrease in epididymal adipose tissue cell size in obese rats, histological analyses of epididymal adipose tissue was performed. As shown in Fig. 5, hematoxylin and eosin (H\&E) staining results for epididymal tissue from each group showed that lipid accumulation significantly increased in the HDF compared with ND group (Fig. 5B). However, the size of the adipocytes in the ATM administration group in the epididymal fat was significantly smaller than in the HFD-fed rats (Fig. 5B), and the size of adipocytes in the epididymal adipose tissue was reduced by $35 \%$ in ATM-treated rats.

\section{Effect of ATM in the fatty lever in indexes in HFD-induced obese rats}


Body weights of HFD-fed rats significantly increased in comparison to control rats after five weeks of feeding initiation. The HFD-fed rats exhibited obesity-related characteristics in terms of body weight, weight gain, and food intake compared to those in the ND group at the end of the study. H\&E staining showed that the number of lipid droplets increased in the liver of rats undergoing HFD-induced obesity. Hepatic steatosis and ballooning degeneration of hepatocytes in the liver tissues were higher in the HFD than the ND group (Fig. 5C). However, ATM administration significantly blocked the effects of HFDinduced hepatic steatosis (Fig. 5C).

\section{Discussion}

Obesity is a medical condition in which abnormal or excessive fat accumulates mainly due to sedentary behavior that is linked to lower health-related quality of life, lack of exercise, and intake of foods rich in fats and oils. Trends in overweight prevalence have been increasing rapidly in children and adults and are associated with serious mortalities, including a high hyperlipidemia incidence, type 2 diabetes mellitus, insulin resistance, heart disease, and fatty liver in addition to various types of cancer and osteoarthritis [1]. The currently available treatment options are not potent enough to prevent obesity permanently, and other effective strategies for weight loss may cause other side effects. Therefore, there is a great demand for long-term use of complementary, alternative and safe and effective medicines to treat this pandemic global obesity problem.

In the present study, we investigated the inhibitory effects of ATM on adipocyte differentiation in 3T3-L1 cell and the anti-obesity ATM activities on HFD-induced obesity rats was investigated by analyzing body and fat pad weights, adipocyte size, and blood biochemical profiles.

ATM is well-known as a functional tree with potential medicinal benefits. Diarylheptanoids [24], rhododendrol glycoside [25], and tannins [26] have been isolated from the genus Acer. Other studies have also demonstrated that ATM is rich in abundant functional compounds, including polyphenols, phenethyl glycosides, and flavonoids, suggesting that these compounds exhibit hepatoprotective activities [20,21]. ATM exhibits potent antiangiogenic activity both in vivo and in vitro [27] and has been shown to be cytotoxic to cancer cells [28].

These results are the first to demonstrate that ATM causes inhibition of differentiation and adipogenesis of 3T3-L1 preadipocytes and lipid accumulation in mature differentiated adipocytes via regulation of adipogenic transcription factor expression and Akt signaling. In order to investigate whether ATM affects adipocyte differentiation, we measured the effects of ATM on lipid accumulation during 3T3-L1 preadipocyte differentiation. Adipogenesis is defined as the process by which preadipocytes differentiate into adipocytes. 3T3-L1 cells are induced to differentiate into adipocytes according to a coordinated program and are one of the most useful cell lines for studying adipogenesis [29]. In the present study, lipid accumulation was measured by Oil Red $O$ staining and the TG assay. Our data demonstrated that ATM treatment caused a reduction in TG levels and a decrease in intracellular lipid content in a dosedependent manner as quantified by Oil Red 0 staining in the cytoplasm of treated 3T3-L1 cells. These 
results demonstrate that ATM blocked adipogenesis during adipocyte differentiation via facilitating an effective decrease in lipid formation and lipid accumulation in 3T3-L1 adipocytes. To our knowledge, this is the first study showing the influence of ATM on lipid accumulation in 3T3-L1 adipocytes.

In line with these findings, we examined the effects of ATM on adipogenesis and lipid accumulation in 3T3-L1 adipocytes via regulation of adipogenic gene expression. Differentiation of preadipocytes is regulated by a complex network of transcription factors, mainly consisting of the C/EBP family and PPARy. This cooperative function helps to maintain each of their own high expression levels. Moreover, adipocytes from mice in which the C/EBPa gene was disrupted showed defects in lipid accumulation [30]. Transgenic mice specifically lacking PPARy in adipose tissue exhibited greatly reduced fat pad sizes [31]. PPARy stimulates adipocytes and induces differentiation, while C/EBPa also supports the adipocyte differentiation process. Therefore, low levels of these adipocyte differentiation-specific genes are important markers of adipocyte inhibition.

In the present study, the treatment with ATM caused a decrease in mRNA expression of C/EBP $\beta, C / E B P a$, and PPARY in 3T3-L1 adipocytes. Western blot analysis showed that C/EBPB, C/EBPa, and PPARY protein levels during adipogenesis of 3T3-L1 preadipocytes demonstrated a dose-dependent reduction. These results show that the ATM treatment caused inhibition of adipocyte differentiation via inhibition of PPARY and C/EBP family-associated adipogenesis signaling, and ATM effects on adipogenic transcription factors involved in adipocyte differentiation in 3T3-L1 cells play a critical role in adipogenesis mediation.

It is well known that adipocyte differentiation is associated with multifunctional cellular pathways and requires sequential regulation of adipogenic and lipogenic genes [32]. PPARY and C/EBPa activate gene expressions, such as aP2, LPL, FAS and others, that are involved in adipogenesis and lipogenesis and participate in creating the adipocyte phenotype $[10,11,33]$.

In the present study, the effects of ATM on the expression of aP2, FAS, and LPL were investigated during 3T3-L1 differentiation. The expression of aP2, FAS, and LPL genes is significantly down-regulated in a dose-dependent manner following ATM treatment. Together, our results demonstrate that ATM led to strong suppression of the expression of critical genes involved in creating and maintaining adipogenesis and lipogenesis via lipid storage and accumulation in 3T3-L1 adipocytes.

Several lines of evidence support the concept that the lipogenic pathway is localized to peroxisomes and is important for endogenous activation of PPARY. PPARy mainly regulates the gene network expression involved in adipogenesis and lipid metabolism [34]. PPARy has a major role in the differentiation of preadipocytes into adipocytes involved in obesity development $[35,36]$. The fact that PPARy null mice complete lack adipose tissue clearly demonstrates that PPARY is essential for adipocyte differentiation [37]. Focusing on lipid metabolism genes, FAS regulates fatty acid synthesis from acetyl- and malonylCoA and via the tricarboxylic acid cycle [38]. Therefore, reduction of FAS via ATM treatment could explain the way in which ATM extracts suppress lipid accumulation. aP2 is highly expressed in adipose tissue and plays a lipid binding protein, which reacts as a key factor in intracellular fatty acid transport and lipid metabolism $[10,11]$. The expression of lipoprotein lipase (LPL) is stimulated by PPARy, thus increasing 
fatty acid uptake into adipocytes [11]. These genes are activated in response to PPARy regulation [10, 11]. In this study, ATM caused a reduction in the expressed level of FAS, LPL, and aP2, suggesting that intracellular fatty acid transport and lipid metabolism are reduced due to low lipid accumulation. The expression levels of those adipogenic and lipogenic-related genes decreased in ATM-treated 3T3-L1 adipocytes. These studies explain ATM-induced inhibition of adipocyte differentiation and lipogenesis in 3T3-L1 preadipocytes. Therefore, ATM reduced adipogenesis and lipid synthesis within differentiated adipocytes at the gene level through inhibition of genes involved in adipocyte lipogenesis and fatty acid transport and synthesis, thus leading to lipogenesis.

Akt kinases play an important role in adipogenesis and glucose transport [39]. Inhibition of Akt activation in fibroblasts displays lack of capability to differentiate preadipocytes into adipocytes, and Akt overexpression results in an increase in glucose uptake and adipocyte differentiation [40]. In the present study, our results demonstrate that ATM caused a significant and concentration-dependent decrease in the phosphorylation of Akt in 3T3-L1 adipocytes. In addition, treatment with ATM caused a marked decreased in phosphorylation of GSK3 $\beta$ during adipocyte differentiation of the 3T3-L1 preadipocytes. It is known that Akt phosphorylation can promote adipocyte differentiation via PPARy up-regulation [41]. The Akt signaling cascade is considered important for adipogenesis as it appears to activate PPARy and C/EBPa during induction of 3T3-L1 adipocyte differentiation [41]. Xu et al. showed that Akt activation enhances an important association between the Akt signaling cascade and transcription factors, PPARY and C/EBPa, in induction of 3T3-L1 adipocyte differentiation [42]. Taken together, these results demonstrate that ATM suppressed adipocyte differentiation and lipid accumulation in 3T3-L1 cells via down-regulation of adipogenic transcription factors and the Akt signaling pathway associated with intracellular lipid accumulation.

Rodent models of HFD-induced obesity have been widely used to investigate human obesity and its related metabolic diseases. Five weeks of an HFD leads to overt obesity in rats and is characterized by body weight gain along with an increase of fat tissue weight. In this animal study, we established that HFD-induced obesity in rats caused an increase in body weight of rats and serum TG and TC levels and a decrease in HDL-C levels. However, our results showed that BW gain was lower in rats administrated the ATM extract. The weights of epididymal and perirenal adipose tissue were also markedly reduced in rats supplemented with ATM, indicating that BW loss is mainly due to decreased fat accumulation in epididymal and perirenal adipose tissues. We also observed that serum TG and TC were lower in rats fed HFD plus ATM than those of rats fed HFD, indicating that ATM is beneficial for improvements in numerous serum metabolic parameters. Thus, these data suggest that ATM suppressed lipid disorders caused by the excess lipid found in HFD-induced obese rats, thereby improving hypertriglyceridemia and hypercholesterolemia caused by HFD-induced obesity. In addition, increased adipose tissue mass, particularly the amount of visceral fat, is associated with an increased risk of metabolic diseases. In this study, ATM-fed rats showed lower masses of epididymal and perirenal adipose tissues. Thus, supplementation with ATM caused reduction in BW and fat tissue weights, thus preventing fat accumulation and improving to obesity. 
Next we investigated the effect of ATM extract for the prevention on fatty liver. The liver is mostly regarded as an essential organ in lipid metabolism. Imbalance between lipid deposition and removal results in hepatic lipid accumulation, which is related to an increase in hepatic lipogenesis, augmented lipid uptake, and/or reduced TG export of $\beta$-oxidation products (43). In the present study, the liver tissue in the obese HFD-fed rats showed lipid droplet accumulation in hepatocytes, while the treatment with ATM caused a decrease in the deposits of hepatic lipids in liver tissue. These results indicate that the livers of ATM-treated rats had less adipocytes than the HFD-fed rats, thus inducing lipid droplet reduction in liver tissue. These findings highlight the critical role of ATM in reducing abnormal lipid accumulation in liver tissue in addition to causing a reduction in BW gain.

\section{Conclusions}

In this study, we showed the inhibitory effects of ATM on adipocyte differentiation. ATM treatment significantly inhibited lipid accumulation and adipocyte differentiation of 3T3-L1 preadipocytes in a dosedependent manner. ATM caused a reduction in the expression of C/EBP $\beta$ and subsequent downregulation of activation of the key transcriptional regulators, C/EBP family and PPARY, in 3T3-L1 adipocytes. Furthermore, ATM caused a reduction in expression of adipogenesis-related factors, such as FAS, aP2, and LPL. We also demonstrated that ATM decreased lipid accumulation and adipogenic gene expression via inhibition of Akt signaling in 3T3-L1 preadipocytes that differentiate into adipocytes. Moreover, the administration of ATM effectively inhibited lipid accumulation in epididymal adipose tissue and liver tissue in HFD-induced obese rats. These results suggest that the anti-obesity effects of ATM result from a decrease in adipogenesis and that ATM has potential use as an anti-obesity agent.

\section{Abbreviations}

ATM: Acer tegmentosum Maxim; DMI: dexamethasone, 3-isobutyl-1-methylxanthine, and insulin mixture; HFD: high-fat diet; C/EBP $\beta$ : CCAAT/enhancer binding protein- $\beta$; C/EBPa: CCAAT/enhancer binding protein-a; PPARY: peroxisome proliferator-activated receptor- $ү ;$ FAS: fatty acid synthase; aP2: adipocyte fatty acid-binding protein-2; LPL: lipoprotein lipase; Akt: protein kinase B; GSK3 $\beta$ : glycogen synthase kinase $3 \beta$; TG: triglyceride; TC: total cholesterol; HDL: high-density lipoprotein; LDL: low-density lipoprotein; HDL-C: high-density lipoprotein-cholesterol; MIX: 3-isobutyl-1-methylxanthine; DEX: dexamethasone

\section{Declarations}

\section{Acknowledgements}

Not applicable

\section{Authors' contributions}


HHC, SJL, SHK, and SHJ performed the experiments. THK, HDK, CKW, and JHC conceived the idea, designed the experiments, and interpreted the experimental results. JHC participated in the study design, data analyses and writing the manuscript. All authors read and approved the final manuscript.

\section{Funding}

This research was supported by Basic Science Research Program through the National Research Foundation of Korea (NRF) funded by the Ministry of Education (no. 2016R1D1A1A09918213).

\section{Availability of data and materials}

All data generated or analysed during this study are included in this published article.

\section{Consent for publication}

Not applicable

\section{Ethics approval and consent to participate}

The experimental procedures were performed in accordance with the animal care guideline of the Gyeongsang National University's institutional animal care and use committee (Approval Number: GNU181231-R0066).

\section{Consent for publication}

Not applicable

\section{Competing interests}

The authors declare that they have no competing interests.

\section{Author details}

1) Institute of Animal Medicine, College of Veterinary Medicine, Gyeongsang National University, Jinju 660-701, Korea

2) Department of Foods and Nutrition, Gyeongsang National University, Jinju, 660-701, Korea.

3) Department of Public Health, Division of Environmental Health Science, New York Medical College, Valhalla, NY 10595, USA

4) Department of Food Science and Biotechnology, Daegu University, Gyungsan 712-714, Korea

\section{References}


1. Grundy SM, Cleeman JI, Daniels SR, Donato KA, Eckel RH, Franklin BA, Gordon DJ, Krauss RM, Savage PJ, Smith SC Jr, Spertus JA, Costa F. American Heart Association; National Heart, Lung, and Blood Institute. Diagnosis and management of the metabolic syndrome: an American Heart Association/National Heart, Lung, and Blood Institute Scientific Statement. Circulation. 2005;112(17):2735-52.

2. Ebbert JO, Jensen MD. Fat depots, free fatty acids, and dyslipidemia. Nutrients. 2013;5(2):498-508.

3. Ormazabal V, Nair S, Elfeky O, Aguayo C, Salomon C, Zuñiga FA. Association between insulin resistance and the development of cardiovascular disease. Cardiovasc Diabetol. 2018;17(1):122.

4. Jeon T, Hwang SG, Hirai S, Matsui T, Yano H, et al. Red yeast rice extracts suppress adipogenesis by down-regulating adipogenic transcription factors and gene expression in 3T3-L1 cells. Life Sci. 2004;12:3195-203.

5. Ross SE, Erickson RL, Gerin I, DeRose PM, Bajnok L, Longo KA, Misek DE, Kuick R, Hanash SM, Atkins KB, Andresen SM, Nebb HI, Madsen L, Kristiansen K, MacDougald OA. Microarray analyses during adipogenesis: understanding the effects of Wnt signaling on adipogenesis and the roles of liver $X$ receptor alpha in adipocyte metabolism. Mol Cell Biol. 2002;22(16):5989-99.

6. Rosen ED, MacDougald OA. Adipocyte differentiation from the inside out. Nat Rev Mol Cell Biol. 2006;7:885-96.

7. Lowe CE, O’Rahilly S, Rochford JJ. Adipogenesis at a glance. J Cell Sci. 2011;124:2681-686.

8. Rosen ED, Walkey CJ, Spiegelman BM.Transcriptional regulation of adipogenesis. Genes Dev. 000;14:1293-307.

9. Tang QQ, Lane MD. Adipogenesis: from stem cell to adipocyte. Annu. Rev. Biochem. 2012;81:715736.

10. Makowski L, Brittingham KC, Reynolds JM, Suttles J, Hotamisligil GS. The fatty acid-binding protein, aP2, coordinates macrophage cholesterol trafficking and inflammatory activity: macrophage expression of aP2 impacts peroxisome proliferator-activated receptor $\mathrm{y}$ and IKB kinase activities. $\mathrm{J}$ Biol Chem. 2005;280(13):12888-12895.

11. Moseti D, Regassa A, Kim WK. Molecular Regulation of Adipogenesis and Potential Anti-Adipogenic Bioactive Molecules. Int J Mol Sci. 2016;17(1):124.

12. Magun R, Burgering BM, Coffer PJ, Pardasani D, Lin Y, Chabot J, Sorisky A. Expression of a constitutively activated form of protein kinase B (c-Akt) in 3T3-L1 preadipose cells causes spontaneous differentiation. Endocrinology. 1996;137:3590-3593.

13. Garofalo RS, Orena SJ, Rafidi K, Torchia AJ, Stock JL, Hildebrandt AL, Coskran T, Black SC, Brees DJ, Wicks JR, et al. Severe diabetes, age-dependent loss of adipose tissue, and mild growth deficiency in mice lacking Akt2/PKBß. J. Clin. Investig. 2003;112:197-208.

14. Green CJ, Göransson O, Alessi DR, Hundal HS. Use of Akt inhibitor and a Drug-resistant Mutant Validates a Critical Role for Akt in the Insulin-dependent Regulation of Glucose and System a Amino Acid Uptake. J. Biol. Chem. 2008;283:27653-27667. 
15. Peng XD, Xu PZ, Crawford ML, Coleman KG, Hay N. Dwarfism, impaired skin development, skeletal muscle atrophy, delayed bone development, and impeded adipogenesis in mice lacking Akt1 and Akt2. Genes Dev. 2003;17:1352-1365.

16. Kohn AD, Summers SA, Birnbaum MJ, Roth RA. Expression of a constitutively active Akt Ser/Thr kinase in 3T3-L1 adipocytes stimulates glucose uptake and glucose transporter 4 translocation. J Biol Chem. 1996;271(49):31372-8.

17. Xu J, Liao K. Protein kinase B/AKT 1 plays a pivotal role in insulin-like growth factor-1 receptor signaling induced 3T3-L1 adipocyte differentiation. J Biol Chem. 2004;279:35914-22.

18. Baudry A, Yang ZZ, Hemmings BA. PKBalpha is required for adipose differentiation of mouse embryonic fibroblasts. J Cell Sci. 2006;119:889-97.

19. Yu T, Lee J, Lee YG, Byeon SE, Kim MH, Sohn EH, Lee YJ, Lee SG, Cho JY. In vitro and in vivo antiinflammatory effects of ethanol extract from Acer tegmentosum. J. Ethnopharmacol. 2010;128:139147.

20. Tung NH, Ding Y, Kim SK, Bae KH, Kim YH. Total peroxyl radical-scavenging capacity of the chemical components from the stems of Acer tegmentosum maxim. J. Agric. Food. Chem. 2008;56:1051010514.

21. Park KM, Yang MC, Lee KH, Kim KR, Choi SU, Lee KR. Cytotoxic phenolic constituents of Acer tegmentosum maxim. Arch. Pharm. Res. 2006;29:1086-1090.

22. Kim S, Hur SJ, Kim KH, Gi KS, Whang WK. Antioxidant and anti-inflammatory compounds isolated from Acer tegmentosum. Journal of Medicinal Plants Research. 2012;6(23):3971-3976.

23. Kim YS, Kang SJ, Kim JW, Cho HR, Moon SB, Kim KY, et al. Effects of Polycan, a $\beta$-glucan, on experimental periodontitis and alveolar bone loss in Sprague-Dawley rats. Journal of Periodontal Research. 201;47(6):800-810.

24. Morikawa T, Tao J, Toguchida I, Matsuda H, Yoshikawa M. Structures of new cyclic diarylheptanoids and inhibitors of nitric oxide production from Japanese folk medicine Acer nikoense. J Nat Prod. 2003;66:86-91.

25. Yonezawa T, Lee JW, Akazawa H, Inagaki M, Cha BY, Nagai K, Yagasaki K, Akihisa T, Woo JT. Osteogenic activity of diphenyl ether-type cyclic diarylheptanoids derived from Acer nikoense. Bioorg Med Chem Lett. 2011;21:3248-3251.

26. Barbehenn RV, Jones CP, Karonen M, Salminen JP. Tannin composition affects the oxidative activities of tree leaves. J Chem Ecol. 2006;32:2235-2251.

27. Kim EC, Kim SH, Piao SJ, Kim TJ, Bae K, Kim HS, Hong SS, Lee BI, Nam M. Antiangiogenic Activity of Acer tegmentosum Maxim Water Extract in Vitro and in Vivo. J Korean Med Sci. 2015;30(7):979-87.

28. Park KM, Yang MC, Lee KH, Kim KR, Choi SU, Lee KR. Cytotoxic phenolic constituents of Acer tegmentosum maxim. Arch Pharm Res. 2006;29:1086-1090.

29. Poulos SP, Dodson MV, Haussmann GJ. "Cell line models for differentiation: preadipocytes and adipocytes.” Experimental Biology and Medicine. 2010;235(10):1185-1193. 
30. Wang ND, Finegold MJ, Bradley A, Ou CN, Abdelsayed SV, Wilde MD, et al. Impaired energy homeostasis in C/EBP alpha knockout mice. Science. 1995;269:1108-1112.

31. He W, Barak Y, Hevener A, Olson P, Liao D, Le J, et al. Adipose-specific peroxisome proliferatoractivated receptor gamma knockout causes insulin resistance in fat and liver but not in muscle. Proc Natl Acad Sci U S A. 2003;100:15712-15717.

32. Gwon SY, Ahn JY, Jung CH, Moon BK, Ha TY. "Shikonin suppresses ERK 1/2 phosphorylation during the early stages of adipocyte differentiation in 3T3-L1 cells." BMC Complementary and Alternative Medicine. 2013;13(1):207.

33. Rosen ED, Walkey CJ, Puigserver P, Spiegelman BM. Transcriptional regulation of adipogenesis. Genes \& Development. 2000;14:1293-1307.

34. Heikkinen S, Auwerx J, Argmann CA. PPARgamma in human and mouse physiology. Biochim Biophys Acta. 2007;1771:999-1013.

35. Tontonoz P, Hu E, Graves RA, Budavari Al, Spiegelman BM. mPPAR gamma 2: tissue-specific regulator of an adipocyte enhancer. Genes Dev. 1994;8:1224-1234.

36. Tontonoz P, Hu E, Spiegelman BM. Stimulation of adipogenesis in fibroblasts by PPAR gamma 2, a lipid-activated transcription factor. Cell. 1994;79:1147-1156.

37. Auwerx J. PPARgamma, the ultimate thrifty gene. Diabetologia. 1999;42:1033-1049.

38. Lodhi IJ, Wei X, Semenkovich CF. Lipoexpediency: de novo lipogenesis as a metabolic signal transmitter. Trends Endocrinol Metab. 2011;22(1):1-8.

39. Kajno E, McGraw TE, Gonzalez E. "Development of a new model system to dissect isoform specific Akt signalling in adipocytes." Biochemical Journal. 2015;468(3):425-434.

40. Zhang HH, Huang J, Düvel K, Boback B, Wu CL, Manning BD. Insulin Stimulates Adipogenesis through the Akt-TSC2-mTORC1 Pathway. PLoS One. 2009;4:e6189.

41. Yoshika D, Sato N, Torisu T, et al. "Adaptor protein SH2-B linking receptor-tyrosine kinase and Akt promotes adipocyte differentiation by regulating peroxisome proliferator-activated receptor $Y$ messenger ribonucleic acid levels." Molecular Endocrinology. 2007;21(5):1120-1131.

42. Xu J, Liao K. Protein kinase B/AKT1 plays a pivotal role in insulin-like growth factor-1 receptor signaling induced 3T3-L1 adipocyte differentiation, J. Biol. Chem. 2004;279:35914-35922.

43. Ipsen DH, Lykkesfeldt J, Tveden-Nyborg P. Molecular mechanisms of hepatic lipid accumulation in non-alcoholic fatty liver disease. Cell Mol Life Sci. 2018;75(18):3313-3327.

\section{Figures}




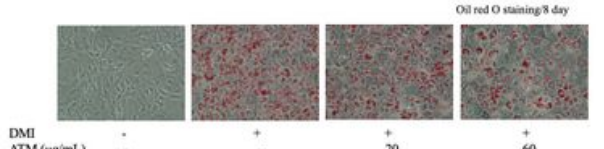

DMI
ATM (pg/mL)

20
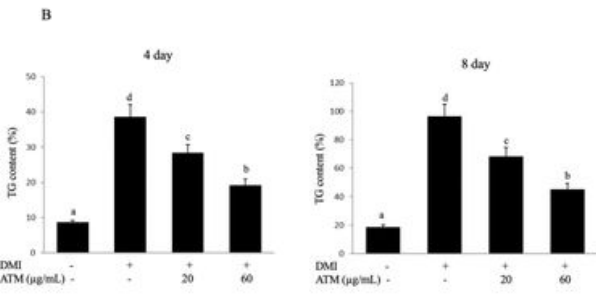
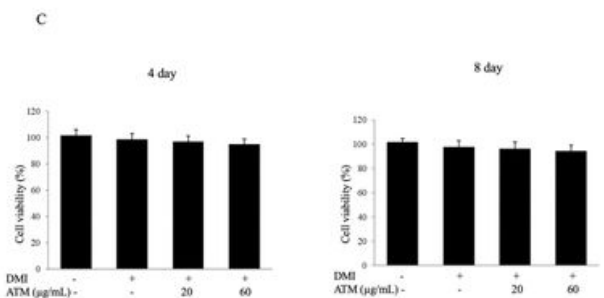

Figure 1

Effects of Acer tegmentosum Maxim (ATM) on lipid accumulation and adipocyte differentiation in 3T3L1 cells. (A) Cellular lipid content was assessed by Oil Red O staining. 3T3-L1 cells were induced to differentiate with desipramine (DMI) and ATM in increasing concentrations $(0,20$, and $60 \mu \mathrm{g} / \mathrm{mL}$ ) and lipid droplets on day 8 after differentiation were measured by Oil Red 0 staining. (B) Triglyceride (TG) content assay of 3T3-L1 cells on days 4 or 8 after the differentiation process. Intracellular TG contents were normalized by total protein. Three independent experiments were used to represent the error bars. The data shown are representative of at least three independent experiments. The values are presented as the means \pm standard deviations (SD). Bars with different letters are significantly different $(p<0.05)$ as determined by Duncan's multiple range test. (C) The viability of 3T3-L1 cells treated with ATM. Cytotoxicity caused by ATM in treated cells was determined by the MTT assay. The values are presented as the means \pm SD. The data shown are representative of at least three independent experiments.
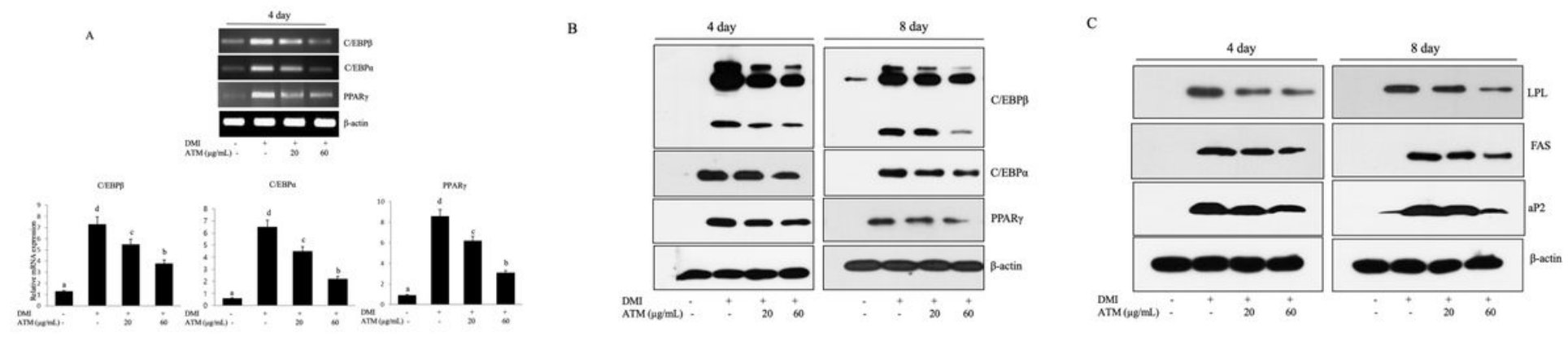

Figure 2

Effects of ATM on adipogenesis-related genes in 3T3-L1 adipocytes. (A) mRNA expression levels of $\mathrm{C} / \mathrm{EBP} \beta, \mathrm{C} / \mathrm{EBPa}$, and PPARy were measured in 3T3-L1 adipocytes on day 4 by reverse-transcriptase polymerase chain reaction (RT-PCR) analysis. Similar results were obtained by three independent experiments. Optical density analysis was performed to quantify the levels of mRNA expression with $\beta$ actin as loading control. (B) The protein expression levels of C/EBP, $\mathrm{C} / \mathrm{EBPa}$, and PPARy were measured in 3T3-L1 adipocytes by Western blot analysis. $\beta$-actin was used as loading control. Bars with different letters are significantly different $(p<0.05)$ as determined by Duncan's multiple range test. (C) Adipogenesis- and lipogenesis-related gene expressions in 3T3-L1 adipocytes on days 4 and 8 as determined by Western blot assay. 

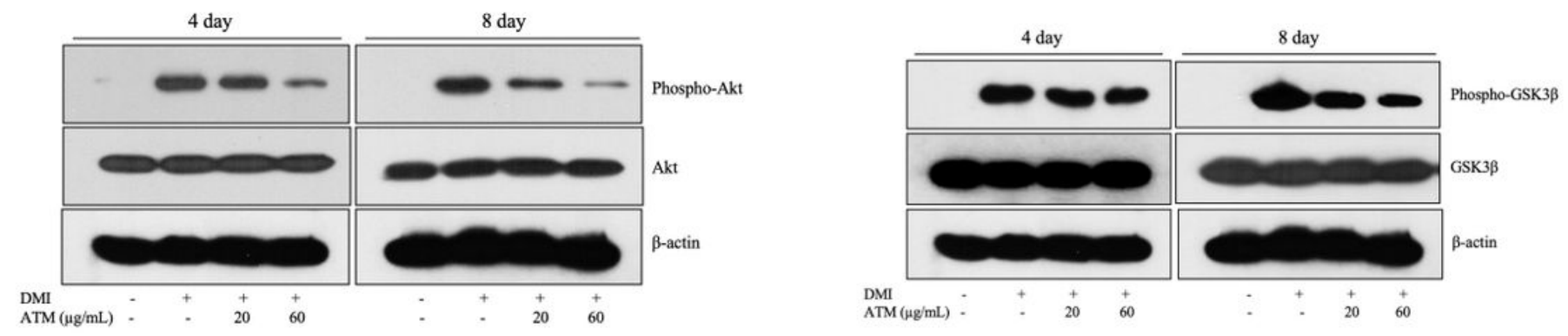

Figure 3

Effects of ATM on the Akt phosphorylation in 3T3-L1 adipocytes. (A) The phosphorylation of Akt in 3T3L1 adipocytes. 3T3-L1 cells were differentiated with DMI in the absence or the presence of ATM for 4 or 8 days. The expression levels of Akt and phospo-Akt were measured in 3T3-L1 adipocytes by Western blot analysis. (B) Phosphorylation of GSK3 $\beta$ in 3T3-L1 adipocytes. 3T3-L1 cells were differentiated with DMI with or without ATM treatment for four or eight days. GSK3 $\beta$ and phospo-GSK3 $\beta$ expression levels were measured in 3T3-L1 adipocytes by Western blot analysis.

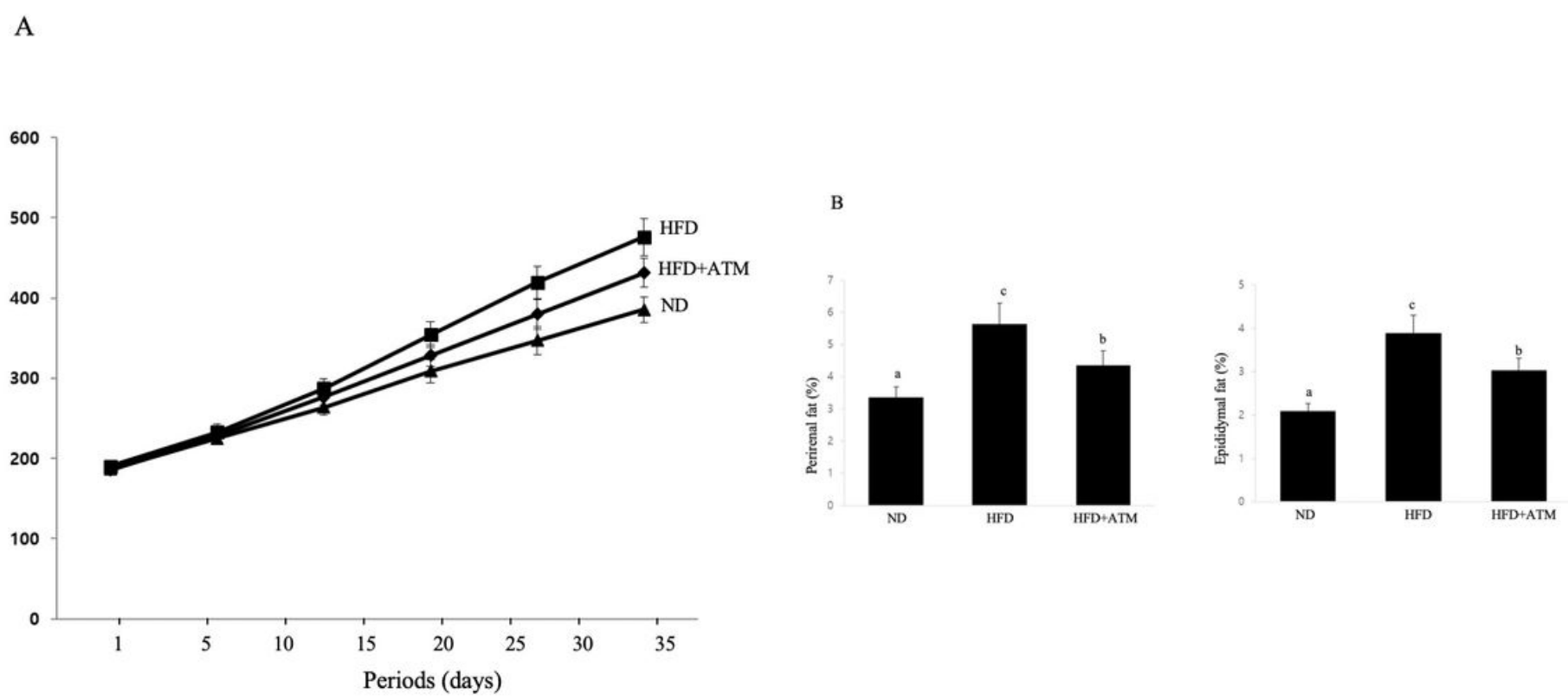

Figure 4

Effects of ATM on adiposity in HFD-induced obese rats. Rats $(n=10)$ were fed a normal diet or high-fat diet (HFD) for five weeks in the presence $(150 \mathrm{mg} / \mathrm{kg} /$ day) or absence of ATM. (A) Effect of ATM on body weight in HFD-induced obesity in rats. The control group was fed a normal diet (ND), HFD+ATM groups were fed an HFD plus ATM (150 mg/kg body weight [BW]), and HFD groups were fed a high-fat diet (diet with $60 \% \mathrm{kcal}$ fat). The BWs of each of the animals were measured twice a week. BWs at the end of the 
experiments were significantly different between the HFD and ND $(P<0.01)$ and HFD+ATM groups $(P<$ 0.05). (B) Effects of ATM on perirenal and epididymal fat weights. The values are presented as means \pm SD. Bars with different letters are significantly different $(p<0.05)$, as determined by Duncan's multiple range test.
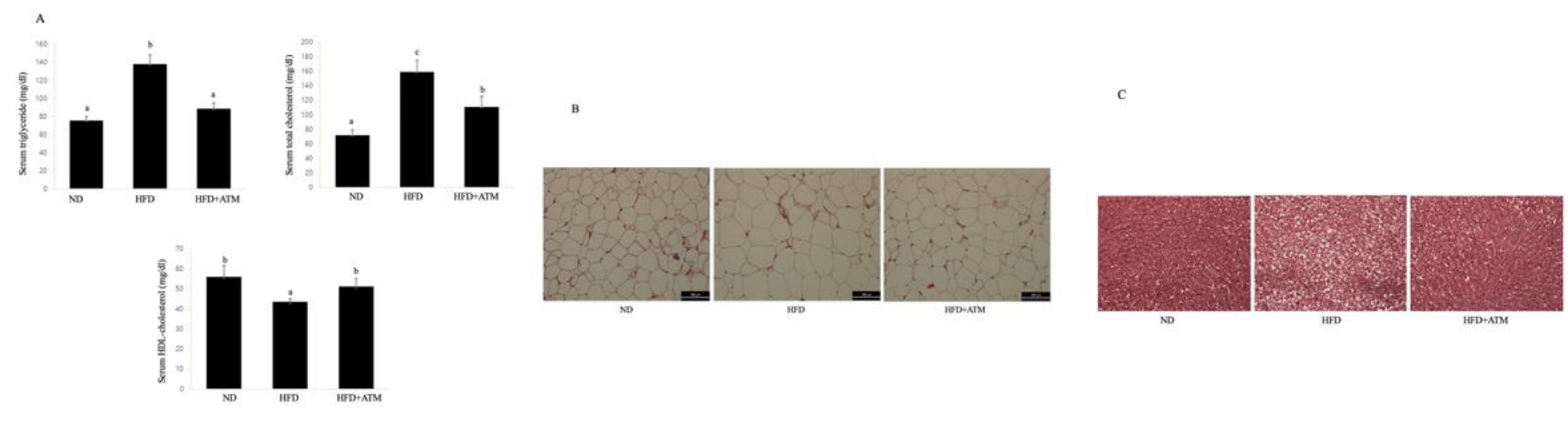

Figure 5

Lipid profiles and histological examination in rats fed with the HFD alone or HFD plus ATM. (A) Effects of ATM treatment on lipid content in HFD-induced obesity in rats. Significant decreases were observed in serum TG and total cholesterol (TC) levels of the ATM-treated group compared to the HFD alone group. HDL-C levels were significantly higher in the ATM-treated group compared to that in the HFD alone group. Values are presented as the means \pm SD. Bars with different letters are significantly different $(p<0.05)$. (B) Effects of ATM on histology of epididymal adipose tissue of rats fed an HFD. Histological analysis of epididymal adipose tissue after staining with hematoxylin and eosin (H\&E) followed by microscopic analysis. Scale bar is $100 \mu \mathrm{m}$. (C) Effects of ATM on lipid accumulation in liver tissues. Histological analysis of liver after staining with H\&E followed by microscopic analysis. 